\title{
A Multi-Objective Model-Based Vertical Handoff Algorithm for Heterogeneous Wireless Networks
}

\section{Shumin Wang}

Central South University https://orcid.org/0000-0002-9542-5436

Honggui Deng ( $\nabla$ denghonggui@csu.edu.cn )

Central South University

\section{Rujing Xiong}

Central South University

Gang Liu

Central South University

Yang Liu

Central South University

Hongmei Liu

Central South University

\section{Research}

Keywords: Vertical handoff, multi-objective optimization, network status value, NSGA-II

Posted Date: September 21st, 2020

DOl: https://doi.org/10.21203/rs.3.rs-47406/v1

License: (c) (i) This work is licensed under a Creative Commons Attribution 4.0 International License. Read Full License

Version of Record: A version of this preprint was published at EURASIP Journal on Wireless Communications and Networking on April 6th, 2021. See the published version at https://doi.org/10.1186/s13638-021-01952-0. 


\title{
RESEARCH
}

\section{A Multi-Objective Model-Based Vertical Handoff Algorithm for Heterogeneous Wireless Networks}

\author{
Shumin Wang ${ }^{1}$, Honggui Deng ${ }^{1}{ }^{*}$, Rujing Xiong ${ }^{1}$, Gang Liu ${ }^{1,2}$, Yang Liu ${ }^{1}$ and Hongmei Liu ${ }^{1}$
}

\footnotetext{
${ }^{*}$ Correspondence:

denghonggui@csu.edu.cn

${ }^{1}$ School of Physics and

Electronics, Central South

University, Lushan South Road,

410083 Changsha, China

Full list of author information is

available at the end of the article
}

\begin{abstract}
An optimized algorithm based on multi-objective optimization model is proposed to solve the problem that existing vertical handoff algorithms do not comprehensively consider the impact of users and the network during handoff process. We build Markov chain model of base station to calculate a more accurate network state. Then a multi-objective optimization model is derived to maximize the value of the network state and the user data receiving rate. The multi-objective genetic algorithm NSGA-II is finally employed to turn the model into a final vertical handoff strategy. The results of the simulation for throughput and blocking rate of networks demonstrate our algorithm significantly increases the system throughput and reduces the blocking rate compared with the existing vertical switching strategy.
\end{abstract}

Keywords: Vertical handoff; multi-objective optimization; network status value; NSGA-II

\section{Introduction}

Vertical handoff is considered a key technology of heterogeneous networks to support mobile users switch between networks of different Radio Access Technologies(RATs). It is designed under the principles of improving the quality of service requirements and system resource utilization. However, an increase in the complexity of heterogeneous network environments and diverse service requirements has increased the design difficulty of vertical handoff algorithms. Therefore, the ability to design an efficient and accurate vertical handoff algorithm that can guarantee the quality of service for users is of great interest to the academic community.

In recent years, a large amount of literature has studied vertical handoff schemes. In $[1,2]$, authors used the traditional signal strength-based handoff algorithm and its improved edition for vertical handoff. This algorithm is simple in design and low in complexity, but its single-attribute decision-making handoff algorithm would lead to a 'Ping-Pong' effect if it was applied for vertical handoff. In a heterogeneous network environment, the characteristics of different access networks are greatly varied, thereby executing handoffs only based on received signal strength cannot accurately enable the terminal to access the optimal network. In [3-5], a multi-attribute vertical handoff algorithm was proposed. This algorithm is simple as well, can be easily implemented, and is more reliable compared with a single-attribute decision-making algorithm. However, it is less flexible and less able to consider the dynamic characteristics of the network, which means the performance of the user and the network cannot reach an optimal state. In References [6-9], although a Markov decision process was introduced to make sure users to have a better access to the network with 
low blocking rate and low latency, the quality of service for users still could not be guaranteed if users' own status' changes were not taken into consideration. References [10-15] studied the multi-attribute intelligent vertical handoff algorithms based on fuzzy logic and artificial neural networks. These algorithms can be applied to effectively improve the accuracy and efficiency of decision-making and reduce the 'Ping-Pong' effect under handoff scenarios where more unique decision factors hard to be quantified were involved. However, the complicated calculations make them unsuitable for terminal devices with limited computing capabilities. Reference [16] built a user-centered multi-objective handoff scheme, the scheme can improve the throughput of the system to a certain extent, but still cannot reflect the true state of the base stations (BSs), and it lacks consideration of coordination among users in design, so that network resources cannot be fully utilized.

To address users' service quality problem, insufficient network status consideration and poor handoff coordination in above vertical handoff algorithms, we propose a vertical handoff algorithm based on a multi-objective optimization model. Firstly, the state values of each network in different states are obtained through the Markov decision process. Then a multi-objective optimization model that maximizes the value of network state and user's data receiving rate is constructed. At last, a multiobjective genetic algorithm is applied to solve the model described above and to obtain the user's optimal handoff strategy. The ratio of this scheme to the existing vertical handoff strategy can significantly improve the system coefficient and lower segmentation rate.

The remainder of this article is organized as follows. In section 2, we derive and solve the function of a multi-objective optimization model. In section 3 , we simulate the performance of the handoff algorithm, and section 4 summarizes this article.

\section{Method}

In this section, we construct a multi-objective optimization model for vertical handoff to solve the problem that existing vertical handoff algorithms do not comprehensively consider the impact of users and the network during handoff process. There are two phases. In the first phase, we obtain status value sequence of each BS by Markov chain model. In the second phase we build a multi-objective optimization model to maximize the value of the network state and the user data receiving rate and then the multi-objective genetic algorithm NSGA-II is used to solve the multiobjective optimization model to obtain the final handoff strategy. Table 1 is the notation of variables for this paper.

\subsection{Pre-Judgment stage}

We assume there are $M$ BSs in the heterogeneous networks, and a total of $N(t)$ users are located under the coverage of the heterogeneous networks at time $t$. For each user $j(j=1,2, \ldots, N(t))$, the received signal strength(RSS) coming from each BS can be calculated. We set the distance from user $j$ to BS $i(i=1,2 \ldots, M)$ at time $t$ as $l_{i j}(t)$. Then the RSS user $j$ receives from the base station $i$ at time $t$ can be calculated as follows:

$$
\operatorname{rss}_{i j}\left(l_{i j}(t)\right)=\rho_{i}-10 * \kappa_{i} \ln \left(l_{i j}(t)\right)+\epsilon_{i}^{2}
$$


Table 1 Notations of variables

\begin{tabular}{ll}
\hline Notations & Total number of base stations. \\
\hline$M$ & A total of users located under the coverage of the heterogeneous networks at time $t$. \\
$l_{i j}$ & The distance from user $j$ to BS $i$. \\
$r s s_{i j}$ & The RSS user $j$ receives from the base station $i$. \\
$\rho_{i}$ & The transmission power of BS $i$. \\
$\kappa_{i}$ & The path loss factor of BS $i$. \\
$\epsilon_{i}^{2}$. & Gaussian white noise of RSS. \\
$q_{i j}$ & The data transmission rate user $j$ obtains from base station $i$. \\
$w_{i}^{\prime}$ & The average bandwidth that each channel of the BS $i$ can be allocated. \\
$D_{j}(t)$ & The interference signal strength of user $j$ at time $t$. \\
$\xi_{i}^{2}$ & The white Gaussian noise of the interference signal strength. \\
$\gamma$ & Basic bandwidth requirement of the user. \\
$u_{-} i s(t)$ & The number of handoff users in the heterogeneous networks. \\
$u_{-} i s_{i}$ & The number of handover users in each base station. \\
$u_{-} n o n_{i}$ & The number of non-handover users in each base station. \\
$\tau$ & User's decision gap. \\
$\lambda_{i}$ & The user arrival rate of the BS $i$ within the decision gap. \\
$s_{i}(t)$ & The status of base station $i$ in the heterogeneous network at time $t$. \\
$b_{\max }$ & The maximum bandwidth requirements for the connection. \\
$b_{\min }$ & The minimum bandwidth requirements for the connection. \\
$d_{\max }$ & The maximum delay required for connection. \\
$d_{\min }$ & The minimum delay required for connection. \\
$c_{i}$ & The number of users served in BS $i$. \\
$\eta_{i}$ & The number of channels of each BS $i$. \\
$\beta$ & The damage factor. \\
\hline
\end{tabular}

where $\rho_{i}$ is the transmission power of BS $i, \kappa_{i}$ is the path loss factor of BS $i$, and $\epsilon_{i}^{2}$ represents the Gaussian white noise that satisfies the Gaussian distribution $\left(0, \sigma_{1}^{2}\right)$. Further, according to Shannon's theorem, the data transmission rate $q_{i j}(t)$ that user $j$ obtains from base station $i$ at time $t$ can be calculated as follows:

$$
q_{i j}(t)=w_{i}^{\prime} \times \log _{2}\left(1+\frac{r s s_{i j}(t)}{D_{j}(t)+\xi_{i}^{2}}\right)
$$

where $w_{i}^{\prime}$ is the average bandwidth that each channel of the BS $i$ can be allocated, $\xi_{i}^{2}$ is the white Gaussian noise satisfying the Gaussian distribution $\left(0, \sigma_{2}^{2}\right)$, and $D_{j}(t)$ is the interference signal strength of user $j$ at time $t$.

During decision process, we need to determine the number of non-handoff users under each BS and the total number of handoff users in the heterogeneous networks in order to determine the network status of each BS more accurately. Here we use the basic bandwidth requirement of the user to determine whether each user requires a handoff. For users who newly entered the coverage of the heterogeneous networks and those have not connected to the BS due to blockage during the last handoff, 
the current actual data transmission rate is 0 , which means they are considered as users who need to be handed over. For users who have connected to the BS, we should further consider whether they are handoff users. When user $j$ is connected to BS $i$ at time $t$, we can get $q_{i j}(t)$ according to Eq.(2). The rules are that when $q_{i j}(t)<\gamma(\gamma$ is basic bandwidth requirement of the user $), j$ needs to be handed over, otherwise user $j$ would continue to be connected to BS $i$. In that way, the number of handoff users $u_{-} i s(t)$ in the heterogeneous networks and the number of non-handover users in each base station $u_{-}$non $_{i}(t)$ will be determined.

For the handoff user in heterogeneous networks, a pre-decision module is required to roughly determine the target BS that the user may access to based on user's data transmission rate. It is assumed that $Q_{j}=\left\{q_{1 j}, q_{2 j}, \ldots, q_{M j}\right\}$ is the data transmission rate set that can be obtained by user $j$ from each BS at time $t$, and user $j$ would select the BS with the highest data transmission rate as target for prehandoff. Then we can obtain pre-target BSs for all users and count the number of users who pre-switched to each BS, set as $u_{-} i s_{i}(t)(i=1,2, \ldots, M)$. We assume the user's decision gap is $\tau$, then the user arrival rate $\lambda_{i}$ of the BS $i$ within the decision gap can be calculated as $\lambda_{i}=u_{-} i s_{i}(t) / \tau$. According to the queuing theory [17], the probability that $k$ users would reach each BS in unit time is:

$$
p_{a}(x=k)=\frac{\left(\lambda_{i}\right)^{k}}{k !} e^{-\lambda_{i}}
$$

When the service rate $\mu_{i}$ of each base station is known, the probability that $k$ users would leave the base station in unit time can be obtained:

$$
p_{b}(x=k)=\frac{\left(\mu_{i}\right)^{k}}{k !} e^{-c_{i} \mu_{i}}
$$

where $c_{i}$ is the number of users served in BS $i, c_{i}=u_{-} n_{n} n_{i}(t)$ represents the number of non-handover users in each BS.

The state of the BS would change as the number of users accessing the base station changes, such as the remaining available bandwidth and network delay of the base station. The status of base stations in the heterogeneous network at time $t$ is represented by set $S(t)$, then $S(t)=\left\{s_{1}(t), s_{2}(t), \ldots, s_{M}(t)\right\}$. At each decision time, the same user can only access one BS, and the status of the base station would change based on the Markov chain. In order to find the state value of each BS in different states, it is necessary to first obtain the instant rewards of different states of each BS and the transition probability between states. The instant reward is determined by the network performance of each BS. The better the performance, the greater the instant reward. Here, the remaining available bandwidth and network delay of the BS are chosen as factors to calculate the instant reward of each state of the BS. The instant reward of $\mathrm{BS} i$ at time $t$ is:

$$
R\left(s_{i}(t), i\right)=w_{b} r_{b}\left(s_{i}(t), i\right)+w_{d} r_{d}\left(s_{i}(t), i\right)
$$


where $r_{b}\left(s_{i}(t), i\right)$ is a value function of the remaining available bandwidth of base station $i$ in state $s_{i}(t)$, and $r_{d}\left(s_{i}(t), i\right)$ is a value function of the network delay of $i$ in state $s_{i}(t)$. The remaining available bandwidth is a benefit parameter and its value function is: $r_{b}\left(s_{i}(t), i\right)=\left(b\left(s_{i}(t), i\right)-b_{\min }\right) /\left(b_{\max }-b_{\min }\right)$, where $b_{\max }$ and $b_{\text {min }}$ are the maximum and minimum bandwidth requirements for the connection, $b\left(s_{i}(t), i\right)$ is the actual remaining bandwidth of the base station $i$ in state $s_{i}(t)$. When $b\left(s_{i}(t), i\right)<b_{\min }, r_{b}\left(s_{i}(t), i\right)=0$, while when $b\left(s_{i}(t), i\right)>b_{\max }$, $r_{b}\left(s_{i}(t), i\right)=1$. The network delay is a loss parameter whose value function is: $r_{d}\left(s_{i}(t), i\right)=\left(d_{\max }-d\left(s_{i}(t), i\right)\right) /\left(d_{\max }-d_{\min }\right)$, where $d_{\max }$ and $d_{\min }$ are maximum and minimum delay required for connection. $d\left(s_{i}(t), i\right)$ is the actual network delay of the base station $i$ in state $s_{i}(t)$. When $d\left(s_{i}(t), i\right)<d_{\text {min }}, r_{d}\left(s_{i}(t), i\right)=1$, whereas when $d\left(s_{i}(t), i\right)>d_{\max }, r_{d}\left(s_{i}(t), i\right)=0 . w_{b}$ and $w_{d}$ are the weights of the remaining available bandwidth and network delay respectively.

The real-time reward reflects network performance of the BS under current state rather than the dynamic changes of the state. Therefore, state value of the BS needs to be further determined according to the state transition probability. The state of the $\mathrm{BS}$ is related to the number of users connected to it, and thus the change in the number of users would lead to changes in the state of the BS. Let $t$ be the time when the decision cycle starts, and $c_{i}$ be the user being served in corresponding BS. Then the probability that a user changes from $c_{i}$ to $c_{n e x t}$ can be calculated by the follows, when $c_{\text {next }}<c_{i}$ :

$$
p\left(c_{i}, c_{\text {next }}\right)=\sum_{n=c_{\text {next }}-c_{i}}^{c_{i}} p_{a}\left(x=n+c_{n e x t}-c_{i}\right) p_{b}(x=n)
$$

when $c_{\text {next }} \geq c_{i}$ :

$$
p\left(c_{i}, c_{n e x t}\right)=\sum_{n=0}^{c_{i}} p_{a}\left(x=n+c_{n e x t}-c_{i}\right) p_{b}(x=n)
$$

where $p\left(c_{i}, c_{\text {next }}\right)$ is the state transition probability of the BS. According to basic bandwidth requirements, we are able to determine that the total number of users who need to make a handover decision at time $t$ is $u_{-} i s(t)$. Considering that all handover users may access to the same base station, it is necessary to calculate the $u_{-} i s(t)$ state values corresponding to each BS. Because the number of accessing channels of each BS is limited, the number of users connected to each BS is limited as well. Suppose that the number of channels of each BS in a heterogeneous network is $\eta_{i}$, then according to Eq.(5), Eq.(6), and Eq.(7), when $u_{-} i s(t)>\eta_{i}$, we can calculate the state value of each state:

$$
V^{\pi}\left(s_{i}(t)\right)= \begin{cases}0 & s_{i}(t)=\left\{\eta_{i}+1, u_{i s(t)}\right\} \\ \sum_{s_{i}(t+1)} \mathrm{p}\left(s_{i}(t), s_{i}(t+1)\right) & \\ \times\left(R\left(s_{i}(t+1), i\right)+\beta \times\left(V^{\pi}\left(s_{i}(t+1)\right)\right)\right) & s_{i}(t)=\left\{1, \eta_{i}\right\}\end{cases}
$$


when $u_{-} i s(t) \leq \eta_{i}$ :

$$
V^{\pi}\left(s_{i}(t)\right)=\sum_{s_{i}(t+1)} \mathrm{p}\left(s_{i}(t), s_{i}(t+1)\right)\left(R\left(s_{i}(t+1), i\right)+\beta *\left(V^{\pi}\left(s_{i}(t+1)\right)\right)\right)
$$

In Eq.(8) and Eq.(9), $V^{\pi}\left(s_{i}(t+1)\right)$ represents the state at the next moment, which has multiple possibilities. The probability that the user would transit to each state is $p\left(s_{i}(t), s_{i}(t+1)\right)=p\left(c_{i}, c_{\text {next }}\right)$. $\beta$ is the damage factor. State values of corresponding states of all base stations can be obtained by using a dynamic programming algorithm.

\subsection{Switching Model Design}

According to Eq.(8) and Eq.(9), we can obtain the state value corresponding to each state of the BS. According to the calculation method of Eq.(5) for the instant reward, it can be known that at the same moment, the BS with the larger state value has better network performance and users tend to access the BS with excellent performance in order to obtain higher bandwidth, lower delay, and reduce the blocking rate of the BS when performing a network handover. However, the handover just based on better state of the network cannot guarantee the service quality. We have to consider changes of user's status as well, such as the user 's maximum transmission rate, which is not only related to the average bandwidth of the base station but also the distance from the user to the BS inferred by Eq.(2). we select the user's data transmission rate to reflect the user's service quality. The greater the data transmission rate, the higher the user's service quality. When designing a handover scheme, users would always choose a BS with high data transmission rate and high status value.

In the same decision cycle, there may be multiple users performing network switching. If performing handover with a single handoff user as the center under traditional handoff schemes, it would be less efficient to utilize the overall resource in heterogeneous network system and the network performance would not reach the optimal state as it lacks coordination between handover users during handoffs. In order to improve the resource utilization efficiency and the network performance, when designing the handoff scheme, handover users should be coordinated to carry out a unified design, and the single-user handoff should be converted into a multi-user coordinated handoff in turn. Firstly, we set the connected BSs of all handover users at time $t$ as a matrix variable $\boldsymbol{\Theta}(t)$ :

$$
\boldsymbol{\Theta}(t)=\left|\begin{array}{cccc}
\theta_{11} & \theta_{12} & \cdots & \theta_{1 u_{-} i s(t)} \\
\theta_{21} & \theta_{22} & \cdots & \theta_{2 u_{-} i s(t)} \\
\vdots & \vdots & \ddots & \vdots \\
\theta_{M 1} & \theta_{M 2} & \cdots & \theta_{M u_{-} i s(t)}
\end{array}\right|
$$

The matrix $\boldsymbol{\Theta}(t)$ reflects the network selection results of all handover users at time t, $M$ is the total number of base stations in the heterogeneous network, and $u_{-} i s(t)$ is the number of handover users at time $t$. Each element in thematrix can only take 1 or 0 , rules as follows: 


$$
\theta_{i j}(t)=\left\{\begin{array}{l}
1 \text { user jis connect to } \mathrm{BS}_{i}, \\
0 \text { user jis } \mathrm{b}=\text { not connect to } \mathrm{BS}{ }_{i}
\end{array}\right.
$$

where $i=1,2, \ldots, M$ and $j=1,2, \ldots, u_{-} i s(t)$.

The $i-t h$ row of the matrix is represented as $\boldsymbol{\Theta}(t)_{((i,:))}$, which reflects the situation where the handover user accesses the base station $i$. The $j-t h$ column of the matrix is represented as $\boldsymbol{\Theta}(t)_{((i,:))}$, reflecting the network selection result of user $j$ at time $t$. Similarly, data transmission rate from each base station for all handover users at decision time t can be calculated by Eq.(1) and Eq.(2) and we can construct a data transmission rate matrix $\boldsymbol{Q}(t)$. Its mathematical form is as follows:

$$
\boldsymbol{Q}(t)=\left|\begin{array}{cccc}
q_{11} & q_{12} & \cdots & q_{1 u_{-} i s(t)} \\
q_{21} & q_{22} & \cdots & q_{2 u_{-} i s(t)} \\
\vdots & \vdots & \ddots & \vdots \\
q_{M 1} & q_{M 2} & \cdots & q_{M u_{-} i s(t)}
\end{array}\right|
$$

After obtaining the data transmission rate matrix that reflects the user service quality requirements, we also need to build a state value matrix that reflects the status of the base station, so that we could know better about the network performance of the BS and the user service quality requirements and thus design a reasonable vertical handover scheme. The state value can be obtained according to Eq.(8) and Eq.(9), and the state value sequence of each BS at time $t$ has $u_{-} i s(t)$ elements, which can also be expressed in matrix form:

$$
\boldsymbol{V}(t)=\left|\begin{array}{cccc}
v_{11} & v_{12} & \cdots & v_{1 u_{-} i s(t)} \\
v_{21} & v_{22} & \cdots & v_{2 u_{-} i s(t)} \\
\vdots & \vdots & \ddots & \vdots \\
v_{M 1} & v_{M 2} & \cdots & v_{M u_{-} i s(t)}
\end{array}\right|
$$

Each row of the matrix $\boldsymbol{V}(t)$ is a state value sequence of a base station, and the state value sequence is arranged in descending order.

In order to maximize the resource utilization of the system and satisfy basic requirements of user's service quality at the same time, we need to follow the principle that maximize the sum of state value and the system throughput when designing vertical handoff algorithm. Let matrix $\boldsymbol{\Theta}(t)$ be a decision variable, considering Eq.(11) and Eq.(12), then the following multi-objective optimization model can be obtained:

$$
\left\{\begin{array}{l}
\text { Maxmize } \sum_{i=1}^{M} \sum_{j=1}^{\left\|\boldsymbol{\Theta}_{(i,:)}(t)\right\|_{1}} \boldsymbol{V}_{i j}(t) \\
\text { Maxmize } \sum_{j=1}^{u-i s(t)} \mathbf{Q}_{(:, j)}(t) * \boldsymbol{\Theta}_{(:, j)}(t)
\end{array}\right.
$$

where $Q_{((:, j))}(t)$ represents the data transmission rate from all BSs to user $j$ at time $t,\left\|\boldsymbol{\Theta}_{((i,:))}(t)\right\|_{1}$ is the $1-$ norm of the vector $\boldsymbol{\Theta}_{((i,:))}(t)$, which represents the total number of users connected to BS $i$ at time $t$. The value of each element in $\Theta$ can only take 0 or 1 . In mathematical form, it can be expressed as: $\theta_{i j}\left(1-\theta_{i j}\right)=$ $0\left(i=1,2, \ldots, M, j=1,2, \ldots, u_{-} i s(t)\right)$. The same user can only access at most one base station at the same time, so $\sum_{i=1}^{M} \theta_{i j}(t) \leq 1$, and since the number of accessing channels of each base station is limited, the number of users connected to each base 
station is also limited. That is $0 \leq\left\|\boldsymbol{\Theta}_{((i,:))}\right\|_{1} \leq \eta_{i}(i=1,2, \ldots, M)$. Equation (13) shows that the vertical handoff decision problem has been transferred into a multi-objective optimization problem. The optimization variable here is a matrix $\boldsymbol{\Theta}$. In order to solve this multi-objective optimization model with constraints, the multi-objective genetic algorithm NSGA- II [18] has to been applied. Algorithm steps are shown in Algorithm 1. Firstly, we initialized the population according to the constraints of the model, and then set the iterative stop condition to perform fast non-dominated sorting, congestion allocation, tournament selection, and elite retention strategy to obtain the final handoff strategy.

Algorithm 1 The framework of multi-objective genetic algorithm NSGA-II for solving multi-objective functions.

Input: The matrix of data transmission rate, $Q(t)$; The matrix of state value sequence, $V(t)$; The total number of handoff users, $u_{-} i s(t)$; The number of non-handover users in each base station, $u_{-} n o n_{i}(t)$; The population size: $N_{\text {pop }}$; Total number of base stations: $M$;

Output: The matrix of the connected BSs of all handover users at time $t, \Theta(t)$

1: Complete population initialization based on the principles: $\theta_{i j}\left(1-\theta_{i j}\right)=0(i=$ $\left.1,2, \ldots, M, j=1,2, \ldots, u_{-} i s(t)\right), \sum_{i=1}^{M} \theta_{i j}(t) \leq 1$ and $0 \leq\left\|\boldsymbol{\Theta}_{((i,:))}\right\|_{1} \leq \eta_{i}(i=$ $1,2, \ldots, M)$. Generate the population $W_{\text {pop }} \subseteq R^{M \times u_{-} i s(t) \times N_{\text {pop }}}$ and the corresponding value $V_{p o p} \subseteq R^{1 \times N_{p o p}}$ of the population ;

2: Using the fast non-dominated sorting module to solve the Pareto rank $F_{p o p} \subseteq$ $R^{1 \times N_{p o p}}$ of individuals in a population, the greater the Pareto level, the closer the corresponding solution is to the pareto optimal solution;

3: Calculate the crowding degree of individuals under each pareto level $D_{\text {pop }} \subseteq$ $R^{1 \times N_{\text {pop }}}$, the greater the congestion, the more diverse the value of the population;

4: According to the order of Pareto rank $F_{p o p} \subseteq R^{1 \times N_{p o p}}$ from high to low and the congestion $D_{\text {pop }} \subseteq R^{1 \times N_{\text {pop }}}$ from large to small, select the parent population for cross mutation to generate a new population $W_{\text {pop }} \subseteq R^{M \times u_{-} i s(t) \times N_{p o p}}$, recalculate the value $V_{\text {pop }} \subseteq R^{1 \times N_{\text {pop }}}, F_{\text {pop }} \subseteq R^{1 \times N_{\text {pop }}}$ and $D_{\text {pop }}$;

5: Repeat step 4 until the maximum number of iterations $n_{\text {iter }}$ is exceeded;

6: Find the maximum value of $V_{p o p} \subseteq R^{1 \times N_{p o p}}$ and return the corresponding individual $\Theta(t)$ in the population $W_{\text {pop }} \subseteq R^{M \times u_{-} i s(t) \times N_{\text {pop }} \text {; }}$

7: return $\Theta(t)$;

\section{Experiments and results}

In order to evaluate the performance of the proposed vertical handoff algorithm, we set up a simulation model in MATLAB 2018a, and compared the proposed algorithm (MBMO) with the vertical handoff algorithm (VHO) based on decision tree (DT-VHO) [4], the VHO based on Multi-Armed Bandit Model (MABA) [9] and the user centered Multi-Objective handoff algorithm (MOS) [16]. We built a heterogeneous network model with three BSs, including 3G, 4G, and 5G BS. Each network was placed in a $510 \mathrm{~m} \times 510 \mathrm{~m}$ matrix space, where $3 \mathrm{G}$ BS was located at coordinate point $(250,510), 4 \mathrm{G}$ BS was located at coordinate point $(-10,0)$, and 
$5 \mathrm{G}$ BS was located at coordinate point $(510,0)$ position. Then we supposed 100 users were randomly generated in a matrix space of $500 \mathrm{~m} \times 500 \mathrm{~m}$ in the middle of the base station. Those users' moving speed was $0 \sim 2 \mathrm{~m} / \mathrm{s}$ and the initial direction of the movement was random. The relevant parameters of the three BSs are given in Table 2 and Common simulation parameters are given in Table 3.

Table 2 Experimental Parameters.

\begin{tabular}{llll}
\hline & $3 \mathrm{G}$ & $4 \mathrm{G}$ & $5 \mathrm{G}$ \\
\hline Coverage radii of BSs & $7 \mathrm{~km}$ & $50 \mathrm{~km}$ & $25 \mathrm{~km}$ \\
Maximum number of serving users in BSs & 10 & 20 & 16 \\
Bandwidths of BSs & $5 \mathrm{MHz}$ & $20 \mathrm{MHz}$ & $24 \mathrm{MHz}$ \\
Transmission powers of BSs & $10 \mathrm{watts}$ & $20 \mathrm{watts}$ & $30 \mathrm{watts}$ \\
Strength of the interfering signal & $-22 \mathrm{dBm}$ & $-8 \mathrm{dBm}$ & $-7 \mathrm{dBm}$ \\
Pass loss factor $\kappa_{i}$ & 0.7 & 1 & 1 \\
Average access bandwidth & $0.5 \mathrm{MHz}$ & $1 \mathrm{MHz}$ & $1.5 \mathrm{MHz}$ \\
Maximum time delay & $300 \mathrm{~ms}$ & $800 \mathrm{~ms}$ & $320 \mathrm{~ms}$ \\
Average time delay & $30 \mathrm{~ms}$ & $40 \mathrm{~ms}$ & $20 \mathrm{~ms}$ \\
\hline
\end{tabular}

Table 3 Common simulation parameters.

\begin{tabular}{ll}
\hline Basic bandwidth requirements of users for BSs: $\gamma$ & $3 M b p s$ \\
Additive white Gaussian noise power of RSS: $\epsilon_{i}^{2}$ & $\epsilon_{i}^{2} \sim N(0,3) d B m$ \\
Additive white Gaussian noise power : $\xi_{i}^{2}$ & $\xi_{i}^{2} \sim N(0,5) d B m$ \\
Time slot: $\tau$ & 2 second \\
\hline
\end{tabular}

\subsection{Analysis of Total Throughput}

Throughput reflects the amount of data transmitted by the system in unit time. The higher the throughput, the higher the transmission efficiency of the system. It is an important performance indicator of the network. Figure 1 shows the throughputs under four types of vertical handoff algorithms MBMO, DT-VHO [4] , MABA [9] and MOS [16] when connected with different numbers of access-users. Among them, the throughput of MBMO is calculated by Eq.(13) introduced in this article. From Fig. 1, we can see that the overall trend of throughput change is that when the number of accessing users is less than 50, the throughput would increase continuously with the increase of users. When the number of accessing users is greater than 50, the throughput would increase slowly and gradually balance. This is because the number of accessing users allowed by the heterogeneous network is limited. When the number of accessing users is greater than 50, the system tends to be saturated. We can also see that the proposed handoff algorithm MBMO always had the highest throughput.

\subsection{Analysis of Blocking Rate}

Figure 2 illustrates how the blocking rate of the four algorithms would change as the number of accessing users increases. The blocking rate curve of the MBMO algorithm is obtained by Eq.(13). It can be seen from the Fig. 2 that overall, the blocking rate would show an ascending trend with the increase in accessing users. 
When the number of accessing users is less than 30, the system still has capacity to accommodate more new users, so the blocking rate of each algorithm is small or even close to 0 . Nevertheless, when the number of accessing users is greater than 30 , the blocking rate of each algorithm would start to increase. With the same number of accessing users, the blocking rate under MOS method is always very large, because it always considers the worst case and overlaps the base stations that all users can access. As a result, this algorithm could not truly reflect the blocking rate of the base station. The benefit function constructed by the DT-VHO method does not consider real-time changes in the base station state during the decision period, so its blocking rate changes gently but could not achieve an optimal effect. Both the MBMO algorithms proposed in this article and the MABA consider the real-time changes of base station state, therefore, the blocking rates under those two methods are always in a low state, but it can be seen from the construction method of Eq.(13) that the proposed MBMO algorithm further considers the coordination between handover users and the quality of service requirements of users and thus would further reduce the blocking rate of the system.

\subsection{Stability of Algorithm}

In order to verify the stability of the proposed algorithm, we simulate the throughput of each handoff user after switching when the number of handoff users is less than the system capacity. The simulation results of each algorithm are shown in Fig. 3. The proposed algorithm MBMO and the vertical handoff algorithm MABA based on the multi-armed bandit model take into account the dynamic characteristics of the networks when users switch, so when the system capacity is sufficient, there will be almost no handoff users cannot access the base station due to a certain base station blocking. The user-centered vertical handover algorithm MOS and the decision tree-based vertical handover algorithm DT-VHO focus on a single user and thus lack the consideration of network-side characteristics, resulting in large fluctuations in the data reception rate obtained by users and unstable user performance. In addition, it can be clearly seen from the mean error graph of the user throughput in Fig. 4 that the MBMO algorithm has the highest mean and the smallest variance. That is because the proposed algorithm considers the performance of both the user side and the network side.

\section{Summary and conclusions}

In this article, we propose a vertical handoff algorithm based on a multi-objective optimization model. We build a multi-objective optimization model with full consideration of the dynamic characteristics of the network side and the quality of service requirements of the user side. In order to quantify the performance of the network state and make it convenient for mathematical processing, a Markov chain is used to solve the value sequence of the BS. Finally, we obtain the decision result by the improved NSGA-II algorithm. Simulation results show that our algorithm could achieve high throughput and low blocking rate so that it guarantees the users' service quality and the overall performance of heterogeneous networks.

Abbreviations

RATs:Radio Access Technologies; BSs:Base stations; RSS:Received signal strength; 
Availability of data and materials

The datasets used and analysed during the current study are available from the corresponding author on reasonable request.

Competing interests

The authors declare that they have no competing interests.

Funding

This research and the writing of this manuscript has not been supported by any funding.

Author's contributions

All the six authors have contributed to the proposal of concept, analysis, design, and algorithm of the paper, and all the authors have read and approved the final manuscript.

\section{Acknowledgements}

Not applicable.

Author details

${ }^{1}$ School of Physics and Electronics, Central South University, Lushan South Road, 410083 Changsha, China.

${ }^{2}$ College of Information Science and Engineering, Changsha Normal University, Teli Road, 410100 Changsha, China.

References

1. Chiussi, F.M., Khotimsky, D.A., Krishnan, S.: Mobility management in third-generation all-ip networks. IEEE Communications Magazine 40(9), 124-135 (2002)

2. Xie, X., Rong, B., Zhang, T., Lei, W.: Improving physical layer multicast by cooperative communications in heterogeneous networks. IEEE Wireless Communications 18(3), 58-63 (2011)

3. Wang, S., Fan, C., Hsu, C., Sun, Q., Yang, F.: A vertical handoff method via self-selection decision tree for internet of vehicles. IEEE Systems Journal 10(3), 1183-1192 (2016)

4. Ma, B., Wang, D., Cheng, S., Xie, X.: Modeling and analysis for vertical handoff based on the decision tree in a heterogeneous vehicle network. IEEE Access 5, 8812-8824 (2017)

5. Yu, H.-W., Zhang, B.: A heterogeneous network selection algorithm based on network attribute and user preference. Ad Hoc Networks 72, 68-80 (2018). doi:10.1016/j.adhoc.2018.01.011

6. Stevens-Navarro, E., Lin, Y., Wong, V.W.S.: An mdp-based vertical handoff decision algorithm for heterogeneous wireless networks. IEEE Transactions on Vehicular Technology 57(2), 1243-1254 (2008)

7. Chen, L., Li, H.: An mdp-based vertical handoff decision algorithm for heterogeneous wireless networks. In: 2016 IEEE Wireless Communications and Networking Conference, pp. 1-6 (2016)

8. Bin, M., Hong, D., Xianzhong, X., Xiaofeng, L.: An optimized vertical handoff algorithm based on markov process in vehicle heterogeneous network. China Communications 12(4), 106-116 (2015)

9. Yang, B., Wang, X., Qian, Z.: A multi-armed bandit model-based vertical handoff algorithm for heterogeneous wireless networks. IEEE Communications Letters 22(10), 2116-2119 (2018)

10. Kustiawan, I., Chi, K.: Handoff decision using a kalman filter and fuzzy logic in heterogeneous wireless networks. IEEE Communications Letters 19(12), 2258-2261 (2015)

11. Kustiawan, I., Liu, C., Hsu, D.F.: Vertical handoff decision using fuzzification and combinatorial fusion. IEEE Communications Letters 21(9), 2089-2092 (2017)

12. Alotaibi, N.M., Alwakeel, S.S.: A neural network based handover management strategy for heterogeneous networks. In: 2015 IEEE 14th International Conference on Machine Learning and Applications (ICMLA) (2015)

13. Ben Zineb, A., Ayadi, M., Tabbane, S.: Qoe-based vertical handover decision management for cognitive networks using ann. In: 2017 Sixth International Conference on Communications and Networking (ComNet), pp. 1-7 (2017)

14. Chen, J., Wang, Y., Li, Y., Wang, E.: Qoe-aware intelligent vertical handoff scheme over heterogeneous wireless access networks. IEEE Access 6, 38285-38293 (2018)

15. Sun, J., Qian, Z., Wang, X., Wang, X.: Es-dqn-based vertical handoff algorithm for heterogeneous wireless networks. IEEE Wireless Communications Letters 9(8), 1327-1330 (2020)

16. Qiang, L., Li, J., Touati, C.: A user centered multi-objective handoff scheme for hybrid $5 \mathrm{~g}$ environments. IEEE Transactions on Emerging Topics in Computing 5(3), 380-390 (2017)

17. Błaszczyszyn, B., Karray, M.K.: Performance analysis of cellular networks with opportunistic scheduling using queueing theory and stochastic geometry. IEEE Transactions on Wireless Communications 18(12), 5952-5966 (2019)

18. Deb, K., Pratap, A., Agarwal, S., Meyarivan, T.: A fast and elitist multiobjective genetic algorithm: Nsga-ii. IEEE Transactions on Evolutionary Computation 6(2), 182-197 (2002)

Figures

Figure 1 Network total throughput.

Figure 2 Blocking probability. 
Figure 3 Througuput of each handoff user.

Figure 4 The mean errorbar of the user throughput. 
Figures

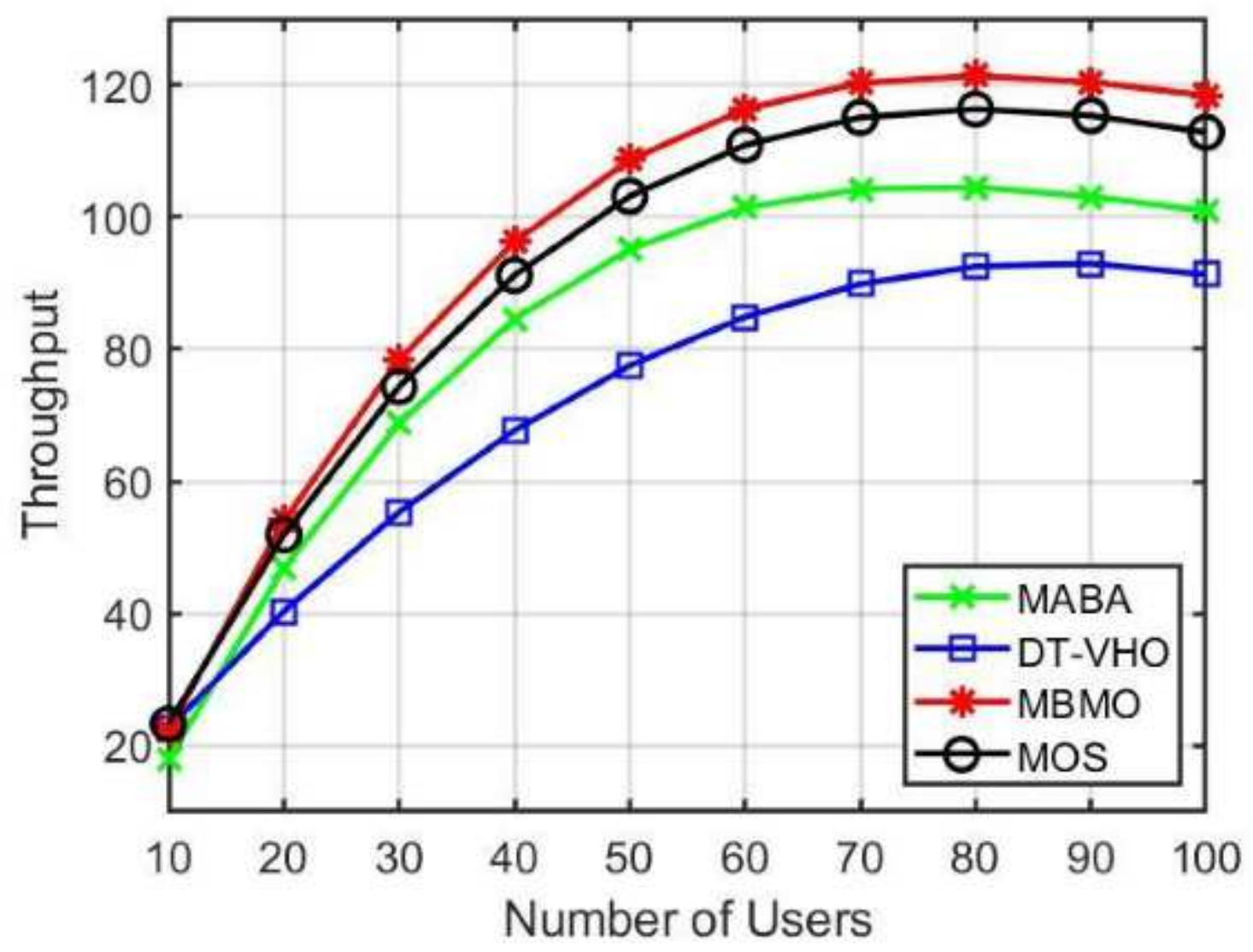

Figure 1

Network total throughput. 


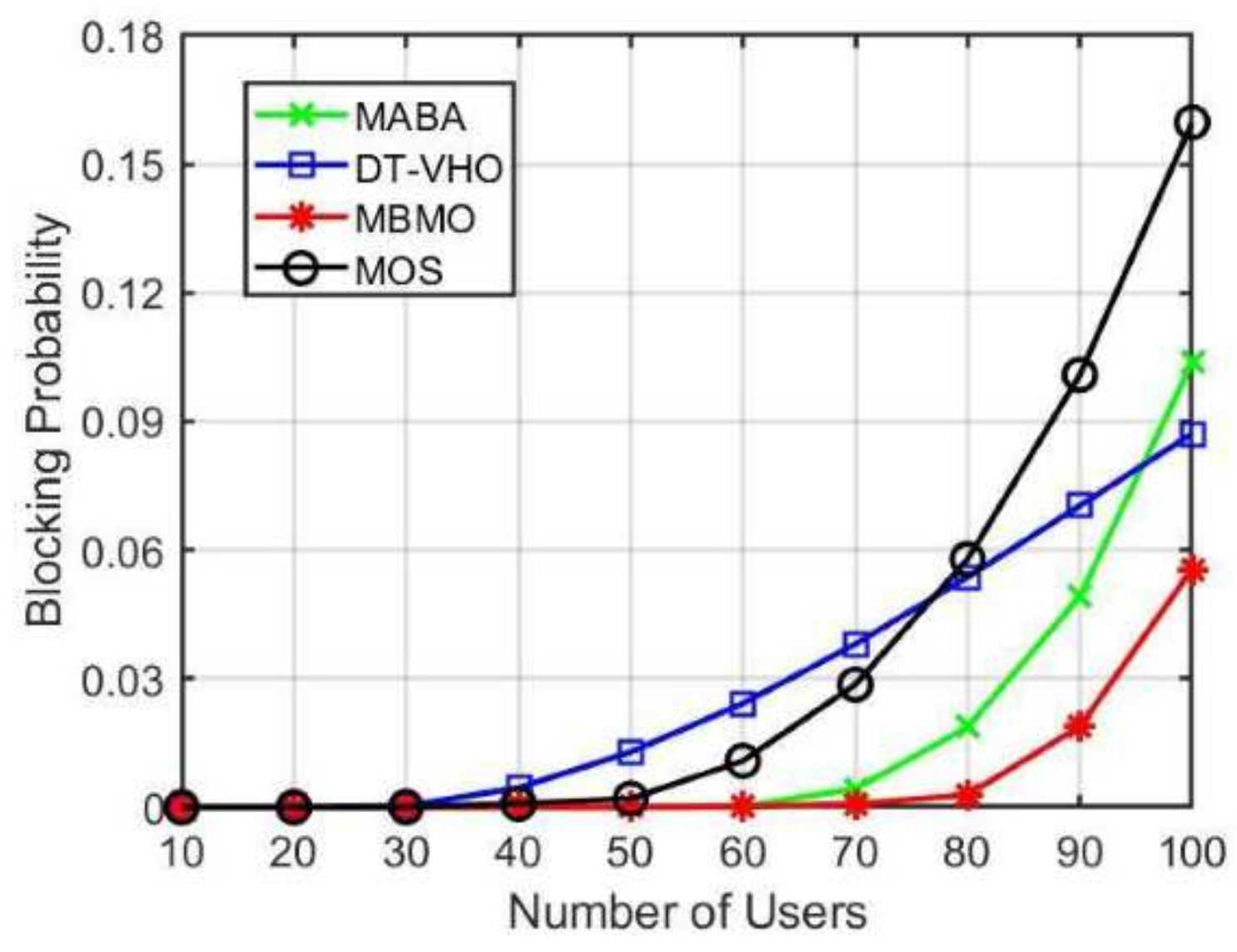

Figure 2

Blocking probability. 

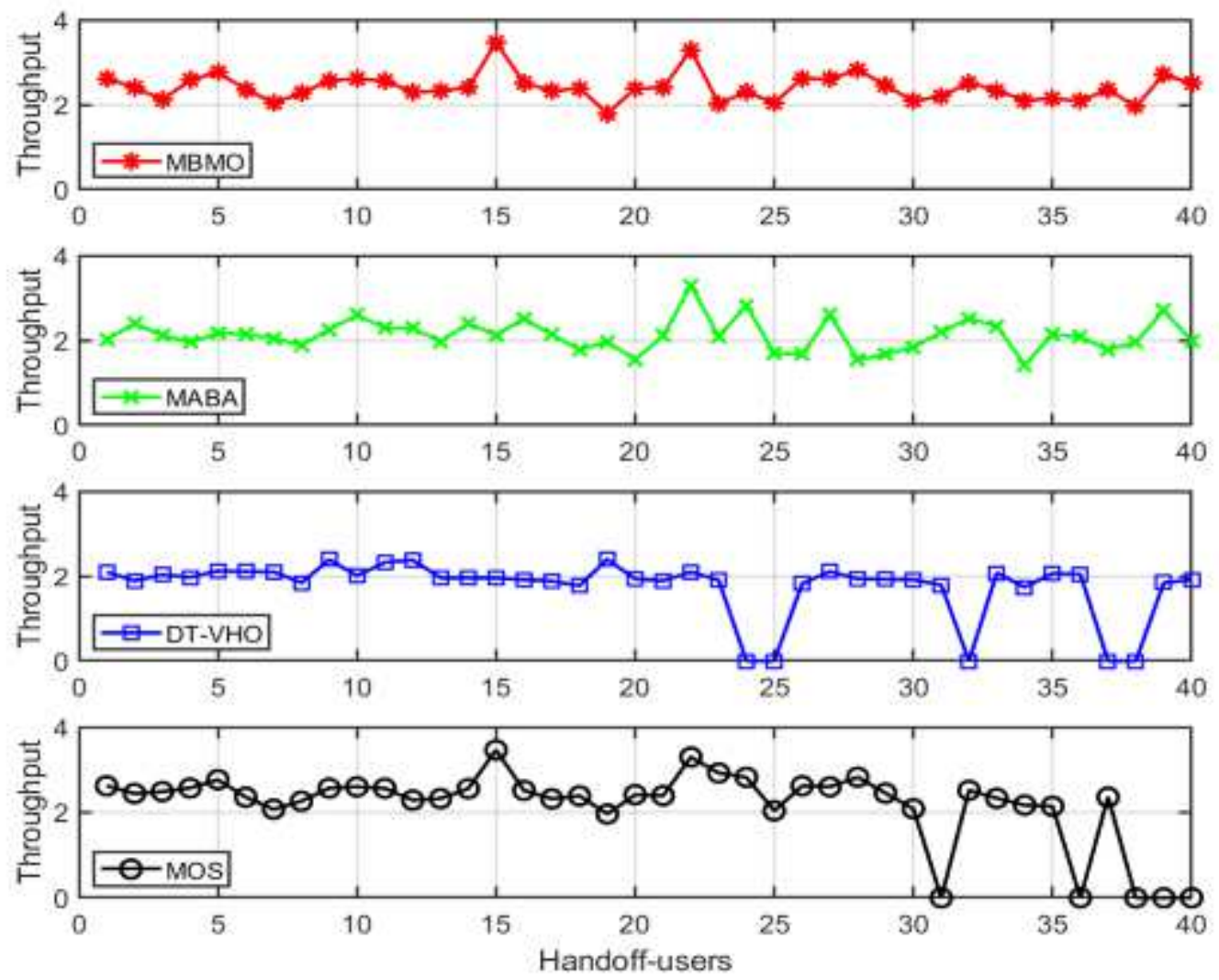

Figure 3

Througuput of each handoff user. 


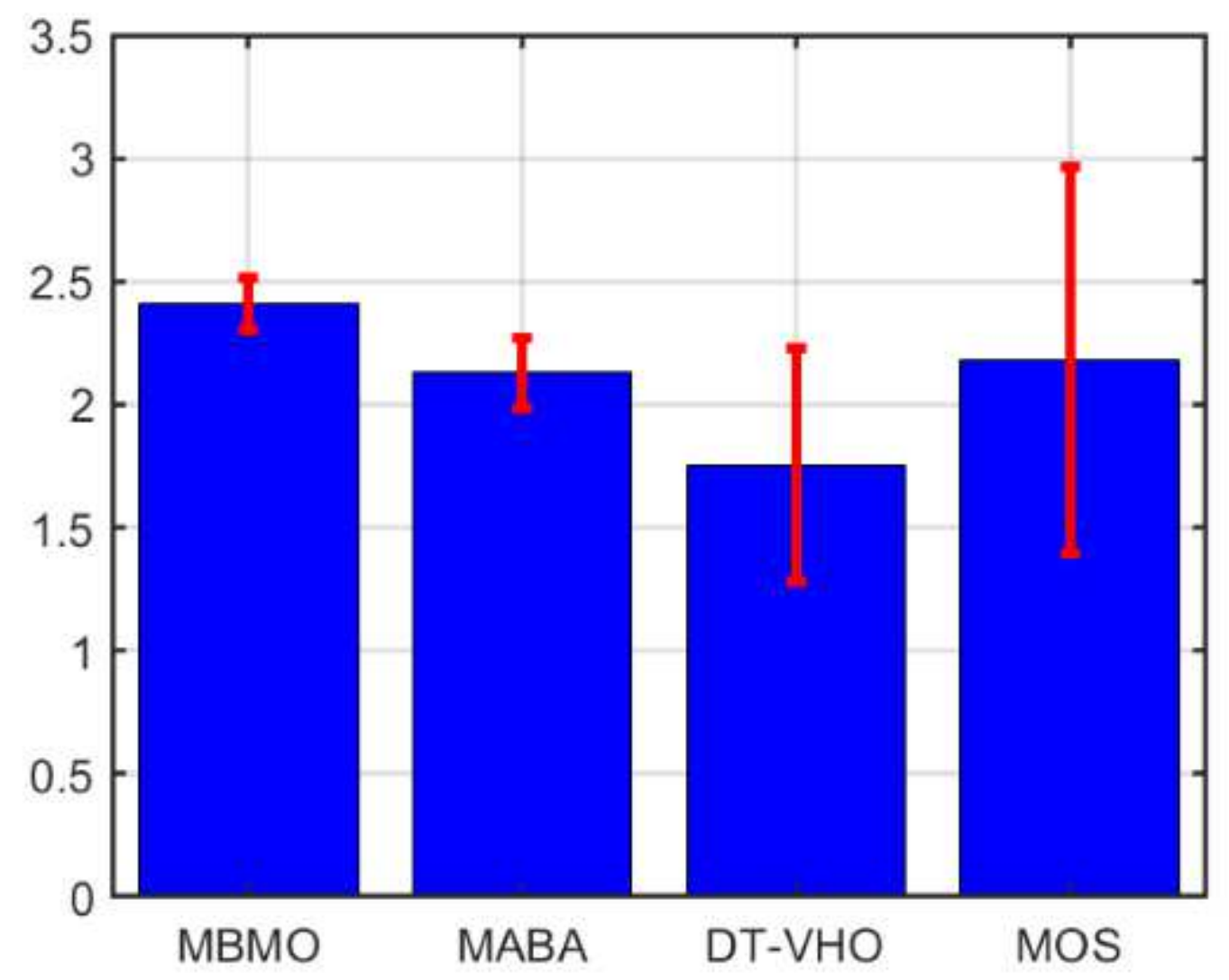

Figure 4

The mean errorbar of the user throughput. 